\title{
Comparative analysis of cognitive maps and mivar nets feasibility in develop decision support systems
}

\section{Chibirova Marina Olegovna}

Russian Federation, Junior Researcher.

Scientific-Research Institute "MIVAR" (SRI "MIVAR"), 127572, Russian Federation, Moscow, Altufevskoe Highway, 95 B. http://www.mivar.ru

\section{mashkache@gmail.com}

\begin{abstract}
The purpose of the study is to research existing approaches in building decision support systems (DSS). Limitations and advantages of utilizing mivar approach and cognitive maps on all stages of DSS design have been analyzed, as well computational complexity of the tasks. Mivar approach has been demonstrated to be a generalization of cognitive maps, which can be considered a special case of mivar nets. Mivar approach justified as a chosen method for simulating, accumulating and logical processing large amounts of data. Choosing mivar approach will improve intelligence of decision support systems, expand boundaries of tasks in hand and build next generation decision support systems.
\end{abstract}

Keywords: mivar, mivar nets, desicion support systems, cognitive maps, cognitive analysis, logical systems with linear computational complexity. 
ISSN 2306-1561

Автоматизация и управление в технических системах (АУТС) 2014. - №1.1(8). - C. 40-54.

DOI: $10.12731 / 2306-1561-2014-1-5$

\title{
УДК 004.8
}

\section{Сравнительный анализ возможностей когнитивных карт и миварных сетей для построения систем поддержки принятия решений}

\author{
Чибирова Марина Олеговна \\ Российская Федерация, младший научный сотрудник. \\ Научно-исследовательский институт «МИВАР» (НИИ «МИВАР»), 127572, Российская \\ Федерация, Москва, Алтуфьевское ш., д. 95 Б. http://www.mivar.ru
}

mashkache@gmail.com

\begin{abstract}
Аннотация. Целью исследования является изучение подходов к созданию систем поддержки принятия решений (СППР). Проанализированы недостатки и достоинства когнитивных карт и миварного подхода на всех этапах разработки СППР, а также вычислительная сложность решаемых задач. Показано, что миварный подход обобщает когнитивные карты, которые могут рассматриваться как частный случай миварных сетей. Обоснован выбор миварного подхода, который позволяет моделировать, накапливать и логически обрабатывать большие объемы данных. Применение миваров позволит повысить интеллектуальность систем поддержки принятия решений, расширить границы рассматриваемых задач и создать системы поддержки принятия решений нового поколения.
\end{abstract}

Ключевые слова: мивар, миварные сети, системы поддержки принятия решений, когнитивные карты, когнитивный анализ, логический вывод с линейной вычислительной сложностью.

\section{1. Введение}

В связи с возрастающей сложностью систем управления становится все более актуальной задача разработки и применения различных программных систем поддержки принятия решения (СППР). СППР представляют собой автоматизированные системы, предназначенные для оказания помощи в принятии решений на основе использования баз данных, документов и моделей областей. Такие системы могут 
опираться на различные методы принятия решений. Можно выделить три основных этапа разработки СППР:

- извлечение и представление знаний;

- принятие решений;

- построение «человеко-машинных» (диалоговых) систем [1].

Задача описания предметной области является первоочередной, т.к. для принятия эффективных решений СППР требуется иметь исчерпывающие знания о рассматриваемой ситуации. На сегодняшний день существуют различные подходы, методы и языки представления данных и знаний. В данной работе проанализируем когнитивные карты, которые получили широкое практическое распространение [1 - 3]. Вместе с тем, известен новый подход, объединяющий многомерное информационное пространство с логической обработкой линейной вычислительной сложности [4 - 28]. Этот подход активно развивается и называется миварные сети (миварный подход).

\section{2. Анализ когнитивного подхода}

Методология когнитивного моделирования предназначена для анализа и принятия решений в слабо структурированных ситуациях, для отображения которых используются когнитивные карты. Когнитивная карта представляет собой ориентированный взвешенный граф (рисунок 1).

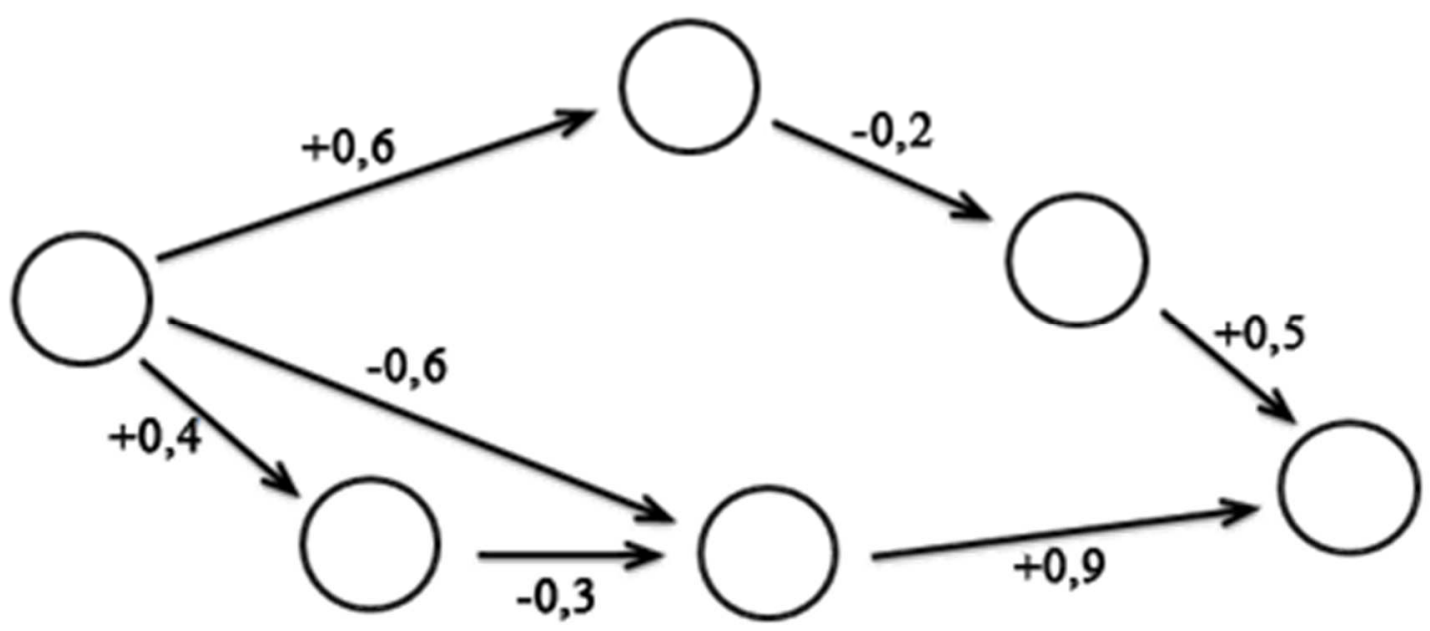

Рисунок 1 - Пример представления когнитивной карты

В нем вершины соответствуют базисным факторам ситуации, в терминах которых описываются процессы в модели. При этом связи определяются между факторами путем рассмотрения причинно-следственных цепочек, описывающих распространение их влияния друг на друга. Стоит отметить, что оно может быть либо усиливающим (положительным), либо тормозящим (отрицательным), либо переменного знака в зависимости от возможных дополнительных условий. При этом необходимо описать шкалу признаков, в соответствии с которой будет определяться вес отношения. В настоящее время для определения степени влияния признаков используется метод 
прямого экспертного назначения силы влияния в виде коэффициента из интервала значений $[-1,1]$ или лингвистического значения из упорядоченного множества, например, \{ «сильно увеличивает»,.., «слабо увеличивает»,.., «не влияет»\}. Данный метод приводит к ошибкам и ограничивает диапазон решаемых задач.

Процесс когнитивного анализа ситуаций состоит из следующих этапов:

- формулировка задачи и цели исследования;

- изучение процесса с позиций поставленной цели;

- сбор, систематизация, анализ существующей статистической и качественной информации по проблеме; источники - СМИ, собственные источники и др.;

- выделение основных характеристических признаков изучаемого процесса и взаимосвязей, определение действия основных объективных законов развития исследуемой ситуации. Это необходимо для нахождения объективных зависимостей, тенденций в процессах;

- определение присущих исследуемой ситуации требований, условий и ограничений;

- определение путей, механизмов действия, реализации интересов основных объектов (субъектов). Это необходимо в дальнейшем для определения стратегии поведения и предотвращения нежелательных последствий развития ситуации [2].

Таким образом, на основе формулировки задачи и цели исследования происходит описание основных элементов ситуации и их характеристик (признаков) в виде каждого элемента $\mathrm{dn}$ и множества признаков $F_{n}=\left\{f_{n} m\right\}, m=1, \ldots, M$. В виде признаков здесь выступают связи одних элементов с другими. Далее экспертным путем определяется когнитивная карта $\left(F_{n}, W_{n}\right)$, являющаяся представлением эксперта о законах функционирования этого элемента, где $W_{n}$ - матрица смежности орграфа размерностью $\mathrm{n} \times \mathrm{n}$, отражающего функциональную структуру элемента ситуации $d_{n}$.

Когнитивные карты отдельных элементов объединяются в общую когнитивную карту:

$$
(F, W)
$$

где $F=\cup F_{n}$ - множество признаков, описывающих ситуацию в целом, а $W$ матрица смежности, включающая матрицы смежности $W_{n}$ отдельных элементов $\mathrm{dn}$ и описывающая их взаимодействие [3].

Перейдем ко второму этапу разработки СППР: принятие решений. В общем виде процесс принятия решений состоит из следующих этапов:

- выявление и диагностика проблемной ситуации;

- постановка цели;

- анализ факторов;

- разработка альтернатив;

- оценивание и выбор альтернатив. 
Анализ текущей ситуации необходим для выявления симптомов проблемной ситуации. Он может быть реализован несколькими методами: интеллектуальный анализ данных, рассуждения на базе прецедентов, нейронные сети, компьютерное имитационное моделирование, OLAP-средства. После выявления проблемы происходит постановка цели, достижение которой может полностью решить проблему. Если в процессе анализа было обнаружено несколько причин возникновения проблемной ситуации, то каждая причина порождает отдельную задачу. После выявления причин проблемных ситуаций и постановки цели рассматриваются задачи выработки альтернатив их решения.

После чего оценивание и выбор альтернатив происходит на основе экспертного мнения или некоторых критериев. При рассмотрении задачи принятия решения с использованием когнитивных карт модель ситуации задается четверкой $\langle F, X, X(0)$, $W)$. Начальными данными является текущее состояние $X(0)=\left(x_{11}^{0}, x_{12}{ }^{0} ., x_{n m}{ }^{0}\right)$, желаемое состояние $X^{G}=\left(x_{11^{g}}, x_{12}{ }^{g}, \ldots, x_{n m}{ }^{g}\right)$ ситуации в функциональной системе поля знаний. Необходимо найти целевой вектор приращений $G=\left(p_{1 j}, p_{2 j}, ., p_{n m}\right)$, где, $p_{11}=x_{11^{g}}{ }^{g} x_{11}{ }^{0}, p_{12}=x_{12}{ }^{g}-x_{12}{ }^{0}$, и т.д. Целевой вектор приращений $G$ показывает, в каком направлении и на сколько нужно изменить значения признаков в начальном состоянии $X(0)$, чтобы перейти в целевое состояние $X^{G}$ [1]. Последовательность таких изменений состояний (переходов) и называют «путем».

Задано множество управляющих признаков $R \subset F$, для каждого из которых определены ограничения на возможные их изменения, т.е. определены ресурсы управления в виде вектора $P R=\left(p_{11}^{r}, \ldots, p_{n m}^{r}\right)$. Задача заключается в нахождении множества решений $U=\left\{U_{1}, U_{2}, \ldots, U_{v}\right\}$ для перевода ситуации из текущего состояния в целевое состояние.

Стоит отметить, что в некоторых случаях важно задание стратегий (алгоритмов) достижения целей. Они определяются экспертами в зависимости от ситуаций и требуют производить либо поиск кратчайшего пути, либо поиск пути с наименьшим или наибольшим весом.

Такие алгоритмы имеют высокую вычислительную сложность и не вариативны. Это также накладывает ограничения на диапазон решаемых задач и полноту их описания (чем больше объем данных, тем дольше происходит поиск решения).

\section{3. Анализ миварного подхода}

Перейдем теперь к способу описания ситуаций с точки зрения миварного подхода, который базируется на эволюционных базах данных и знаний [4 - 6]. Важными достоинствами миварного подхода являются линейный матричный метод поиска логического вывода на адаптивной сети правил $[7,8]$ и эволюционное миварное пространство [9] с возможностью параллельной обработки данных [10, 11]. Миварный подход применяется для решения самых различных задач, включая самоорганизующиеся комплексы диагностики [4, 12], управление мобильными 
роботами [13], создание интеллектуальных систем [14, 15, 30], информационная безопасность [16], распознавание образов [17] и др. Теоретически обоснованы [18, 19] и практически реализованы некоторые миварные интеллектуальные системы [20 - 29]. Отметим, что направление создания миварных систем активно развивается, например, только в 2013 году опубликовано более 8 научных статей [22 - 29].

Миварное пространство представляет собой множество осей, множество элементов осей, множество точек пространства и множество значений точек. Введем $A=\left\{a_{n}\right\}, n=1 . . N$, где $A-$ множество названий осей миварного пространства, $N$ количество осей миварного пространства $[4,5]$. Тогда

$$
\forall a_{n} \exists F_{n}=\left\{f_{n_{i_{n}}}\right\}, n=1 . . N, i_{n}=1 . . I_{n},
$$

где $F_{n}$ - множество элементов оси an,in- идентификатор элемента множества $F_{n}$, $I_{n}=\left|F_{n}\right|$. Множества $F_{n}$ образуют многомерное пространство: $M=F_{1} \times F_{2} \times \ldots \times F_{n}$. $m=\left(i_{1}, i_{2}, \ldots, i_{N}\right), m \in M, m$ - точка многомерного пространства, $\left(i_{1}, i_{2}, \ldots, i_{N}\right)$ координаты точки $m$.

Существует множество значений точек многомерного пространства $M$ :

$$
C_{M}=\left\{c_{i 1}, i 2, \ldots, i N \mid i_{1}=1 \ldots I_{1}, i_{2}=1 \ldots I_{2}, \ldots, i_{n}=1 \ldots I_{N}\right\}
$$

где $c_{i 1, i 2, ., i N}$ - значение точки многомерного пространства $M$ с координатами $c_{i 1, i 2, ., i N}$. Для каждой точки пространства $M$ не существует или существует единственное значение из множества $C_{M}$. Таким образом, $C_{M}$ - множество изменений состояний модели данных, представляемой в многомерном пространстве. Для перехода между многомерным пространством и множеством значений точек введено отношение $\mu: C_{x}=\mu\left(M_{x}\right)$, где $M_{x} \subseteq M, M_{x}=F_{1 x} \times F_{2 x} \times \ldots \times F_{N x}$.

Для описания модели данных в миварном пространстве необходимо выделить три оси: ось отношений $O$, ось признаков (свойств) элемента $S$ и ось элементов (объектов) предметной области $V$. Данные множества являются независимыми.

В многомерном пространстве каждому значению атрибута кортежа отношения соответствует точка с определёнными координатами. Отношения связывают элементы пространства. Множество всех точек многомерного пространства соответствует модели данных. При миварном подходе ее структура определяется точками пространства, которые хранят соответствующие значения атрибутов отношения. Миварная сеть может быть представлена в виде двудольного графа (рисунок 2). 


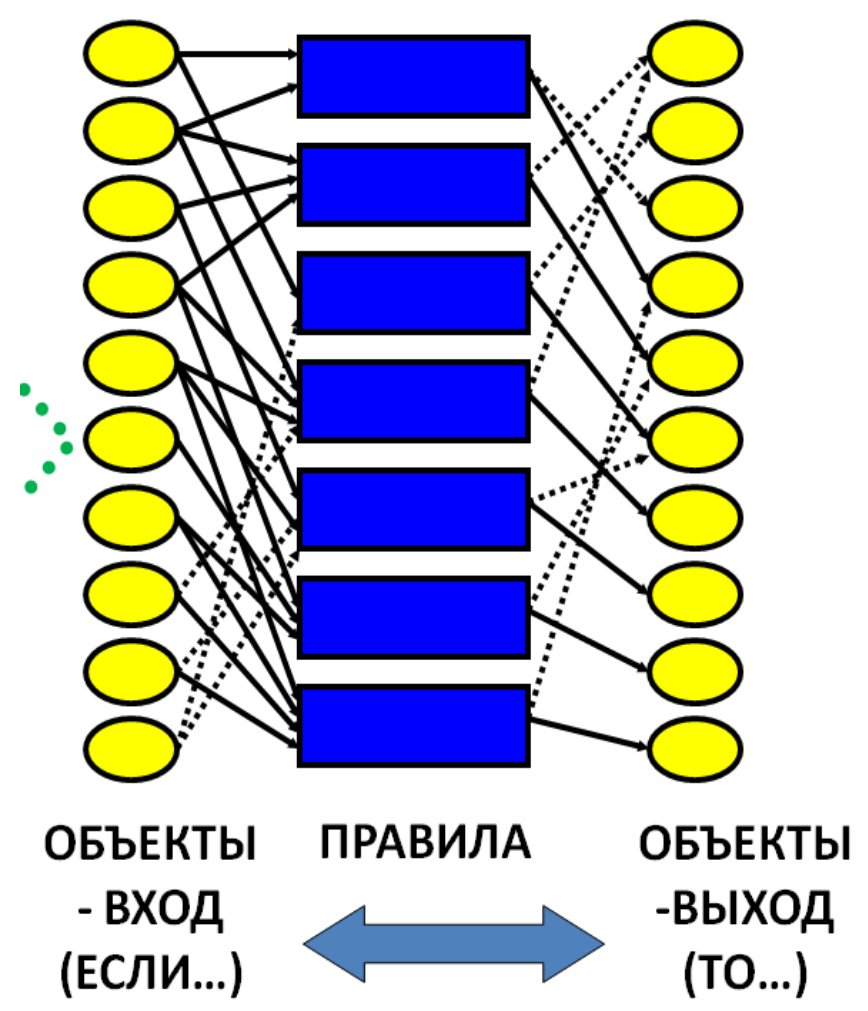

Рисунок 2 - Двудольный граф представления миварной сети

Аналогично с когнитивными картами (1), миварное пространство можно выразить кортежем вида

$$
<V, S, O,>
$$

Стоит отметить, что элементы миварного пространства могут делиться на ряд классов:

- видовые и родовые;

- понятия части и целого;

- понятия элементов многоуровневой иерархии;

- единичные и общие;

- конкретные и абстрактные.

Отношения же могут быть представлены в любом виде: формулы, логические переходы, текстовые выражения, функции, сервисы и т.д. При этом в отношениях не ограничено количество входных и выходных параметров, что обеспечивает полноту описания связей между элементами области и возможность учесть все факторы, способные повлиять на ситуацию.

Процесс анализа и построения миварной модели предметной области состоит из следующих этапов:

- сбор существующих данных в рассматриваемой области;

- выделение и описание объектов, их признаков и отношений.

Предметная область с точки зрения миварного подхода представляется в виде многомерного (базового трехмерного) пространства (рисунок 3). 


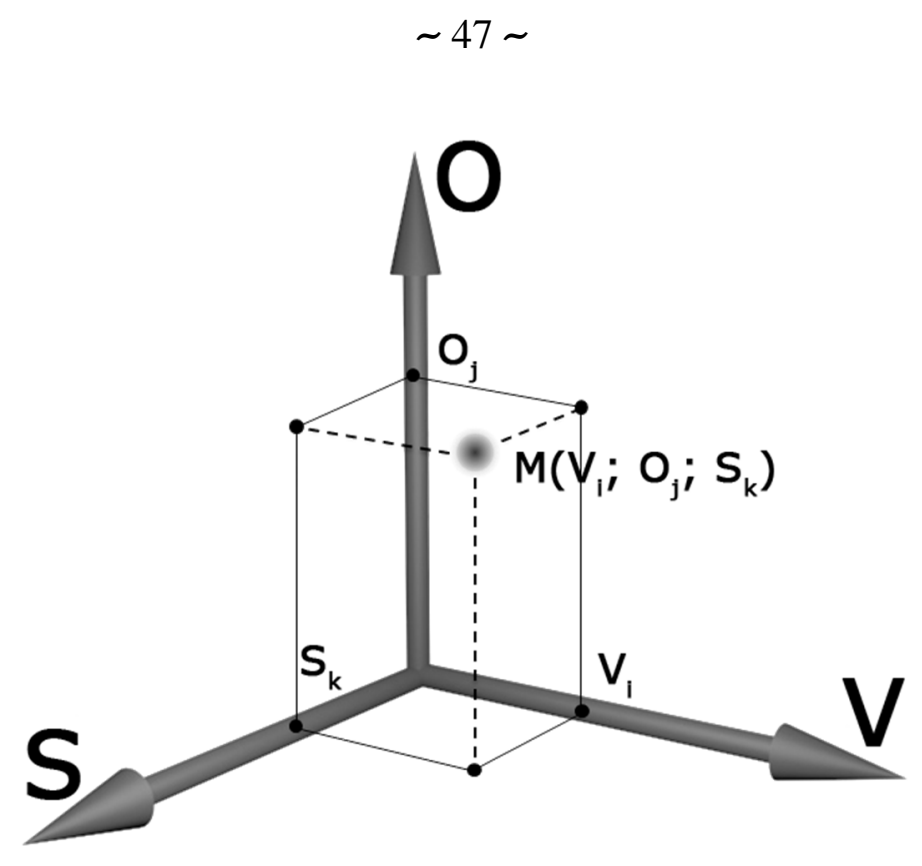

Рисунок 3 - Трехмерное миварное пространство представления данных

В качестве осей выбираются: ось объектов, ось свойств объекта и ось отношений. Ограничения на количество элементов отсутствует, что позволяет наиболее полно описывать предметную область. И элементы, и отношения могут иметь свое неограниченное число свойств. Отношения, свойства и элементы могу образовывать внутри себя множества, классы, иерархии.

Теперь рассмотрим процесс принятия решений на основе миварного подхода. Пусть известны $\mathrm{m}$ - правил и $\mathrm{n}$ - переменных (входящих в правила либо в качестве исходных, активизирующих, либо в качестве получаемых, т.е. выходных переменных). Тогда в матрице $V(n \times m)$ могут быть представлены все взаимосвязи между правилами и переменными. При этом, на каждой строке при описании каждого правила все входные переменные этого правила на соответствующих позициях матрицы помечаются символом х, все выходные - у, все переменные, которые уже получили в процессе вывода или задания исходных данных некоторое конкретное значение - $z$, a все искомые (выходные) переменные, т.е. те, которые необходимо "вывести" из исходных (входных) данных - $w$. Кроме того, добавим в матрицу $V$ одну строку и один столбец для хранения в них служебной информации $[4,8]$.

Тогда, мы получаем матрицу $V$ размерности $(n+1) \times(m+1)$, в которой отражена вся структура исходной сети правил. Сразу отметим, что, как и выше было показано, структура этой логической сети может изменяться в любое время, т.е. это сеть правил с изменяемой (эволюционной) структурой. Схематичное изображение приведено на рисунке 4.

Миварный подход обрабатывает большие объемы данных в режиме реального времени, что позволяет наиболее полно описывать различные предметные области. Решения уникальны для каждой конкретной ситуации и строятся автоматически, без необходимости участия эксперта. 
$\sim 48 \sim$

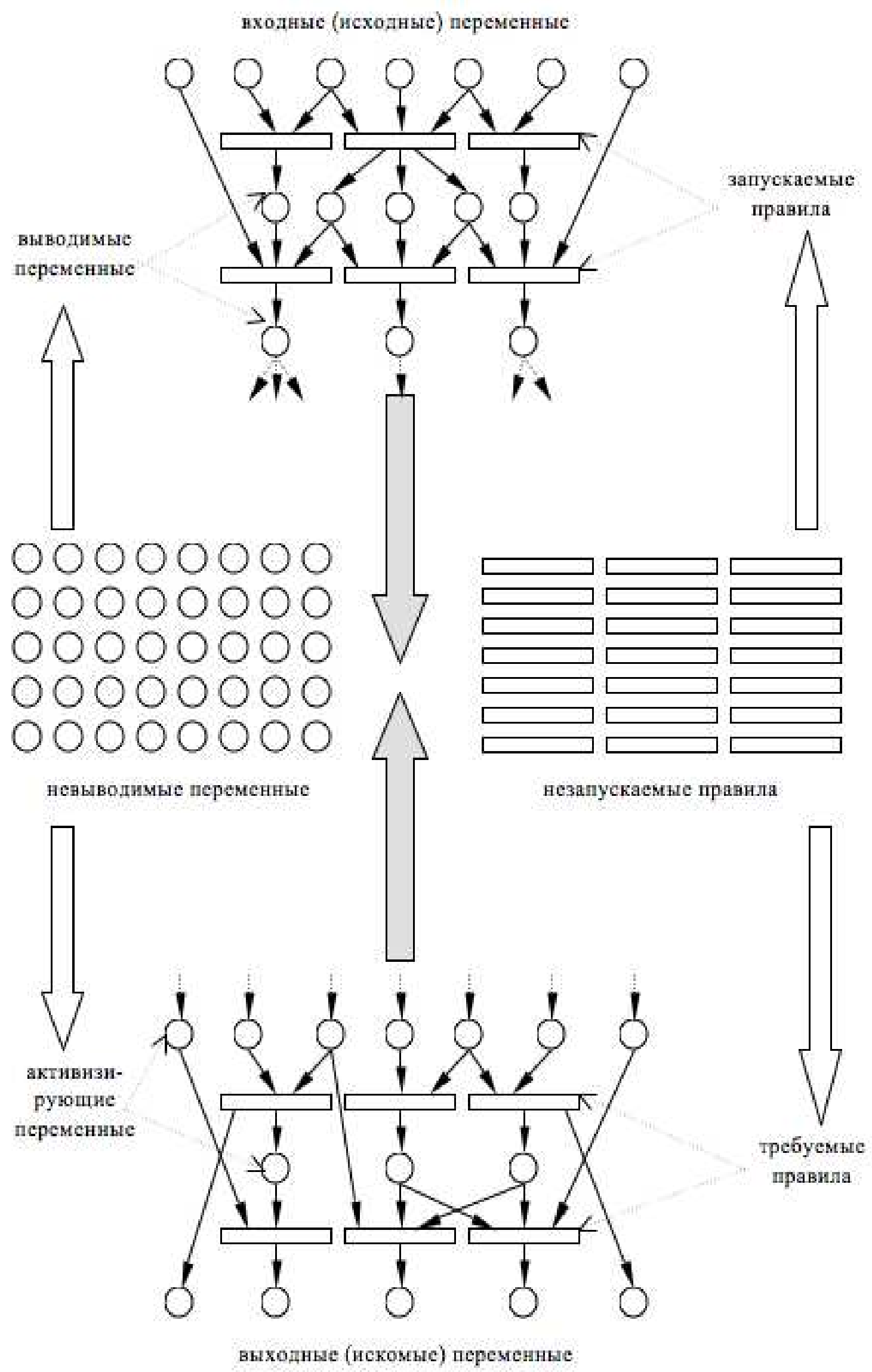

Рисунок 4 - Общее представление алгоритма миварного поиска 


\section{4. Сравнение когнитивных карт и миварного подхода}

Для проведения сравнения разных подходов необходимо, прежде всего, разработать их описание в аналогичных формализмах. Для этого введем следующие обозначения:

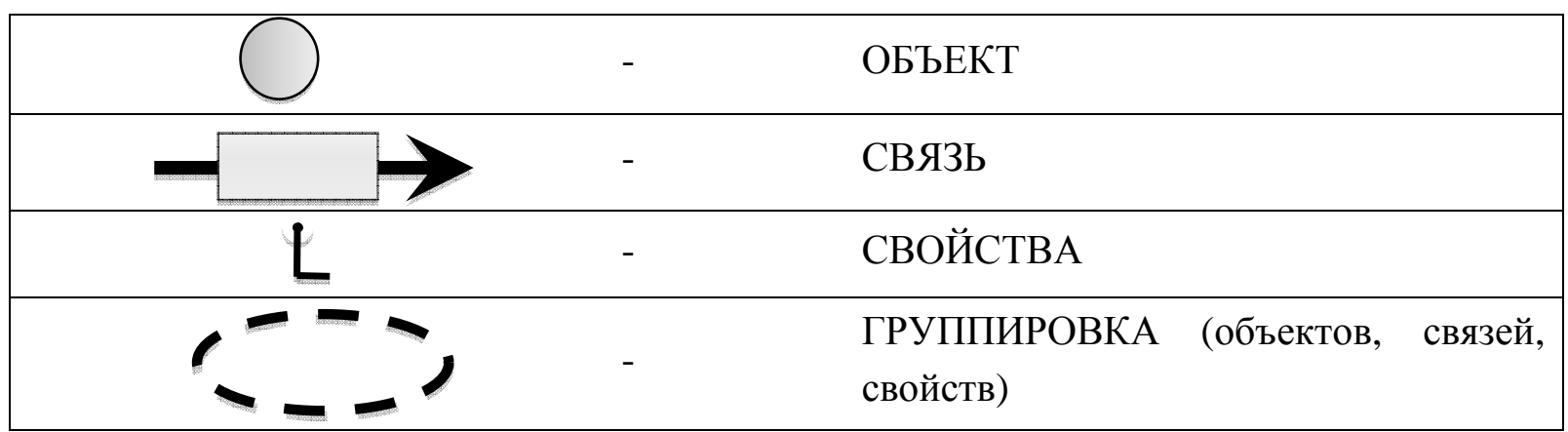

Тогда в общем виде модель когнитивной карты можно представить в виде графа (рисунок 5), на котором связи могут иметь одно свойство, отражающее влияние одного фактора (объекта) на другой.

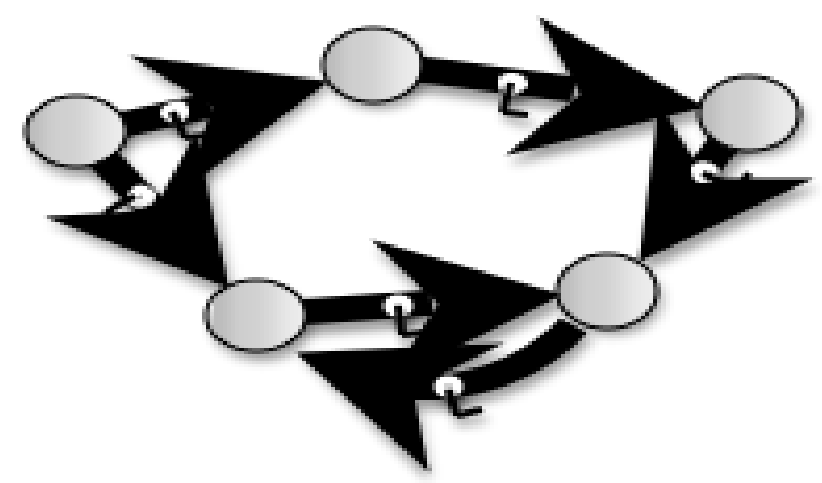

Рисунок 5 - Схематичное представление когнитивного подхода

Представить миварный подход можно следующим образом:

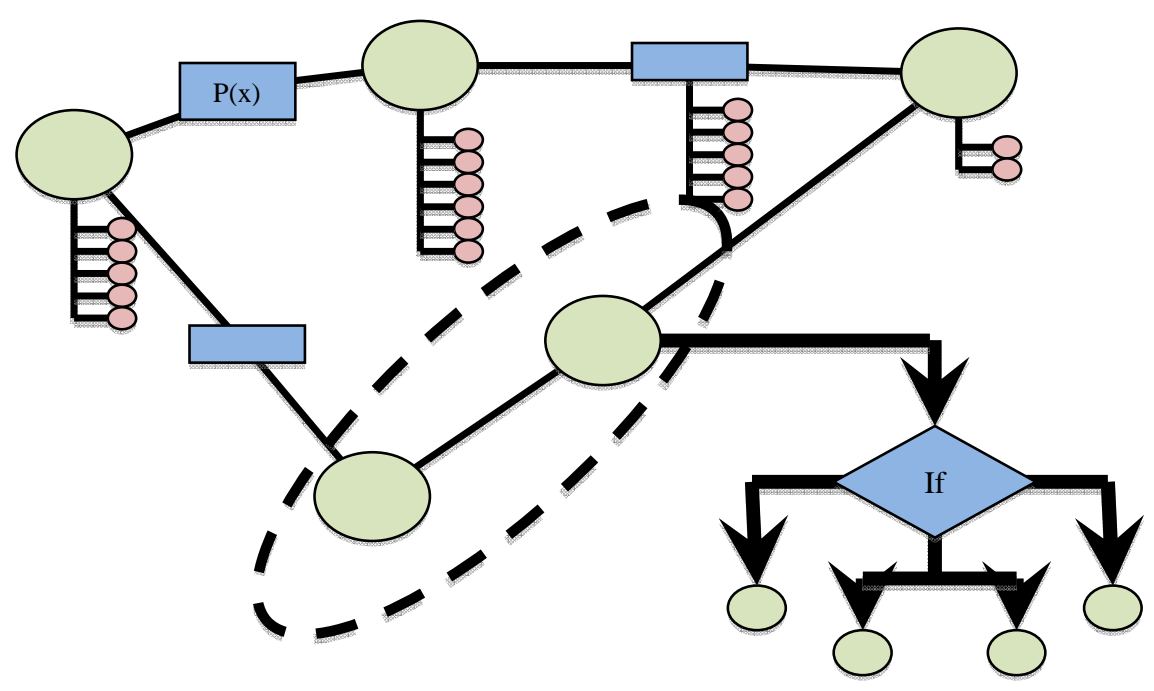

Рисунок 6 - Схематичное представление миварного подхода 
В миварном подходе объекты и связи могут иметь неограниченное количество свойств. Отношения между элементами представляют собой структуру, способную учитывать все необходимое количество объектов, участвующих в ситуации [4 - 29]. Приведем результаты сравнительного анализа в виде таблицы (Таблица 1).

Таблица 1 - Результаты сравнительного анализа различных подходов

\begin{tabular}{|c|c|c|}
\hline Параметры сравнения & Когнитивный подход & Миварный подход \\
\hline $\begin{array}{l}\text { Предварительная } \\
\text { задачи и цели исследования }\end{array}$ & Требуется & Не требуется \\
\hline $\begin{array}{l}\text { Предварительное изучения ситуации с } \\
\text { точки зрения поставленной цели }\end{array}$ & Требуется & Не требуется \\
\hline Сбор данных & $\begin{array}{l}\text { С учетом ограничений } \\
\text { контекста } \\
\text { рассматриваемой } \\
\text { ситуации }\end{array}$ & $\begin{array}{l}\text { Без учета ограничений } \\
\text { контекста } \\
\text { рассматриваемой } \\
\text { ситуации }\end{array}$ \\
\hline Объективность полученной модели & $F$ & + \\
\hline $\begin{array}{l}\text { Возможность описания } \\
\text { элементов (их свойств) }\end{array}$ & Отсутствует & Есть \\
\hline $\begin{array}{l}\text { Возможность описания признаков у } \\
\text { отношений }\end{array}$ & Отсутствует & Есть \\
\hline $\begin{array}{l}\text { Предварительный } \quad \text { анализ } \\
\text { формулировки путей решения }\end{array}$ & Требуется & Не требуется \\
\hline Добавление данных & $\begin{array}{lr}\text { Добавление } & \text { элемента } \\
\text { может } & \text { потребовать } \\
\text { изменение } & \text { структуры } \\
\text { сети или } & \text { изменение } \\
\text { значений } & \text { связей } \\
\text { элементов } & \\
\end{array}$ & $\begin{array}{l}\text { Добавление нового } \\
\text { элемента приводит к } \\
\text { автоматическому } \\
\text { изменению множества, } \\
\text { определяющего ось } \\
\text { пространства }\end{array}$ \\
\hline Изменение данных & $\begin{array}{l}\text { Возможно появление } \\
\text { необходимости } \\
\text { перестраивать модель }\end{array}$ & $\begin{array}{l}\text { Результатом выполнения } \\
\text { является сохранение } \\
\text { значений новых точек в } \\
\text { многомерном } \\
\text { пространстве }\end{array}$ \\
\hline $\begin{array}{l}\text { Описание } \\
\text { элементами }\end{array}$ & $\begin{array}{l}\text { Отношения бинарные } \\
\text { (связана каждая пара } \\
\text { элементов), } \\
\text { весом }\end{array}$ & $\begin{array}{l}\text { Широкие возможности по } \\
\text { описанию } \\
\text { взаимодействия объектов: } \\
\text { формулы, логические } \\
\text { переходы, текстовые } \\
\text { выражения (а так же } \\
\text { внешние сервисы) }\end{array}$ \\
\hline
\end{tabular}




\begin{tabular}{|c|c|c|}
\hline Параметры сравнения & Когнитивный подход & Миварный подход \\
\hline $\begin{array}{lr}\text { Возможность } & \text { вариативного } \\
\text { представления } & \text { данных } \\
\text { иерархическое, общее и т.д.) }\end{array}$ & Отсутствует & Есть \\
\hline $\begin{array}{l}\text { Последующее изучение } \\
\text { модели, описанной } \\
\text { реальности обобщенных понятий }\end{array}$ & Требуется & Не требуется \\
\hline Алгоритм процесса принятия решений & $\begin{array}{l}\text { Задается экспертом (на } \\
\text { этапе описания задачи } \\
\text { или в зависимости от } \\
\text { необходимой цели в } \\
\text { момент решения) }\end{array}$ & $\begin{array}{l}\text { Разрабатывается каждый } \\
\text { раз автоматически }\end{array}$ \\
\hline Вариативность алгоритма & Не вариативен & Вариативен \\
\hline Время выполнения поиска пути & $\begin{array}{l}\text { Экспоненциально } \\
\text { возрастает при } \\
\text { увеличении количества } \\
\text { рассматриваемых } \\
\text { элементов }\end{array}$ & $\begin{array}{l}\text { Выполняется в режиме } \\
\text { реального времени }\end{array}$ \\
\hline
\end{tabular}

Таким образом, видно, что в миварном подходе можно описать и выполнить все, что есть в когнитивных картах. Дополнительно к этому, в миварном подходе есть целый ряд дополнительных преимуществ. Следовательно, можно говорить следующее: миварный подход обобщает и развивает когнитивные карты. А когнитивные карты могут рассматриваться как частный случай миварных сетей.

\section{5. Заключение}

В статье проводился анализ использования двух подходов к разработке СППР. Процесс принятия решения был разделен на несколько этапов, на каждом из которых проводилось сравнение когнитивных карт и миварного подхода.

При использовании когнитивных карт как основу для СППР необходимо:

- предварительно изучить ситуацию в разрезе поставленной задачи;

- описать предметную область с учетом ограничений количества объектов, видов бинарных связей между ними и возможности задания признаков объекта;

- сформулировать алгоритмы (графовые) поиска решений;

- провести изучение описанной модели, определить реальность обобщенных понятий;

- в случае добавления или изменений данных может потребоваться перестроение модели с участием эксперта. 
Такие модели отражают субъективное видение эксперта, описывают конкретную ситуацию с учетом различных ограничений. Они сложно изменяемые и не могут подробно отразить рассматриваемую область.

Использование миварного подхода при разработке СППР не накладывает дополнительных ограничений, необходимо только описать существующие знания в данной предметной области. В этом случае выделенные объекты могут образовывать иерархии, объединяться в классы, наследовать свои отношения и свойства. Отношения в миварной модели могут быть выражены в виде формул, логических переходов, текстовых выражений, функций. При добавление данных происходит автоматическое изменение множеств, определяющих оси пространства, а при изменении сохраняется значение новых точек в пространстве. В ходе решения сам миварный движок генерирует уникальные алгоритмы поиска пути в зависимости от исходных данных. После этого происходит расчёт необходимых выражений (значений) в режиме реального времени и вывод найденного алгоритма с отображением использованных объектов и связей между ними.

Таким образом, миварный подход способен помочь в решении широкого спектра задач, наиболее полно описать предметную область и учесть ее особенности, объяснить правильность выбранного алгоритма и произвести расчет, если это необходимо. Когнитивные карты являются частным и упрощенным случаем миварных сетей и миварного подхода.

\section{Список информационных источников}

[1] Минин Д.В., Фролов С.В. Применение систем поддержки принятия решений при разработке медицинский программно-аппаратных комплексов. [Электронный pecypc]. URL: rgu-penza.ru/mni/content/files/ 10_1_Minin,Frolov.pdf (дата обращения: 06.12.2013).

[2] Максимов В.И., Корноушенко Е.К., Качаев С.В. Когнитивные технологии для поддержки принятия управленческих решений // Информационное общество, 1999, вып. 2, с. 50 - 54.

[3] Кулинич А.А. Разработка принципов и методов построения программных систем поддержки принятия решений в слабо структурированных ситуациях на основе моделирования знаний эксперта// Автореф. дис. канд. тех. наук / Институт проблем управления им.Трапезникова. М., 2003.

[4] Варламов О.О. Эволюционные базы данных и знаний для адаптивного синтеза интеллектуальных систем. Миварное информационное пространство. - М.: Радио и связь, 2002. - 288 С.

[5] Варламов О.О. Системный анализ и синтез моделей данных и методы обработки информации в самоорганизующихся комплексах оперативной диагностики: диссертация на соискание ученой степени доктора технических наук. - М.: МАРТИТ, 2003. 307 с.

[6] Варламов О.О. Обзор 25 лет развития миварного подхода к разработке интеллектуальных систем и создания искусственного интеллекта // Труды НИИР. 2011. № 1. C. 34-44. 
[7] Варламов О.О. Разработка линейного матричного метода определения маршрута логического вывода на адаптивной сети правил // Известия вузов. Электроника. 2002. № 6. C. 43-51.

[8] Варламов О.О. Разработка адаптивного механизма логического вывода на эволюционной интерактивной сети гиперправил с мультиактивизаторами, управляемой потоком данных // Искусственный интеллект. 2002. № 3. С. 363-370.

[9] Варламов О.О. Основы многомерного информационного развивающегося (миварного) пространства представления данных и правил// Информационные технологии, 2003. № 5. С. 42-47.

[10] Варламов О.О. Разработка метода распараллеливания потокового множественного доступа к общей базе данных в условиях недопущения взаимного искажения данных // Информационные технологии. 2003. №1. С. 20-28.

[11] Варламов О.О. Параллельная обработка потоков информации на основе виртуальных потоковых баз данных // Известия высших учебных заведений. Электроника. 2003. № 5. С. 82-89.

[12] Варламов О.О. Системный анализ и синтез моделей данных и методы обработки информации для создания самоорганизующихся комплексов оперативной диагностики // Искусственный интеллект. 2003. № 3. С. 299-305.

[13] Варламов О.О. Системы обработки информации и взаимодействие групп мобильных роботов на основе миварного информационного пространства // Искусственный интеллект. 2004. № 4. С. 695-700.

[14] Варламов О.О. Создание интеллектуальных систем на основе взаимодействия миварного информационного пространства и сервисно-ориентированной архитектуры // Искусственный интеллект. 2005. № 3. С. 13-17.

[15] Варламов O.O. Анализ взаимосвязей GRID и CAC ИВК, SOA и миварного подхода // Искусственный интеллект. 2005. № 4. С. 4-11.

[16] Варламов О.О. О системном подходе к созданию модели компьютерных угроз и еe роли в обеспечении безопасности информации в ключевых системах информационной инфраструктуры // Известия Таганрогского государственного радиотехнического университета. 2006. Т. 62. № 7. С. 216-223.

[17] Максимова А.Ю., Варламов О.О. Миварная экспертная система для распознавания образов на основе нечеткой классификации и моделирования различных предметных областей с автоматизированным расширением контекста // Известия Южного федерального университета. Технические науки. 2011. № 12. С. 77-87.

[18] Варламов О.О. О необходимости перехода от теории искусственного интеллекта к разработке теории активного отражения // Известия Южного федерального университета. Технические науки. 2007. Т. 77. № 2. С. 89-95.

[19] Варламов О.О. О возможности создания интеллектуальных систем на основе GRID, систем адаптивного синтеза ИВК, сервисно-ориентированной архитектуры и миварного информационного пространства // Известия Таганрогского государственного радиотехнического университета. 2005. Т. 54. № 10. С. 130-140.

[20] Владимиров А.Н., Варламов О.О., Носов А.В., Потапова Т.С. Программный комплекс "УДАВ": практическая реализация активного обучаемого логического вывода с линейной вычислительной сложностью на основе миварной сети правил // Труды НИИР. 2010. Т. 1. С. 108-116.

[21] Варламов О.О. Миварные технологии: переход от продукций к двудольным миварным сетям и практическая реализация автоматического конструктора алгоритмов, управляемого потоком входных данных и обрабатывающего более 
трех миллионов продукционных правил // Искусственный интеллект. 2012. № 4. C. 11-33.

[22] Варламов О.О. Практическая реализация линейной вычислительной сложности логического вывода на правилах "ЕСЛИ-ТО" в миварных сетях и обработка более трех миллионов правил // Автоматизация и управление в технических системах. 2013. - № 1(3); [Электронный pecypc]. URL: http://auts.esrae.ru/3-66 (дата обращения: 26.03.2013).

[23] Варламов О.О., Чибирова М.О., Сергушин Г.С., Елисеев Д.В. "Облачная" реализация миварного универсального решателя задач на основе адаптивного активного логического вывода с линейной сложностью относительно правил "Если-То-Иначе" // Автоматизация и управление в технических системах. - 2013. - № 2(4). C. 7-23. [Электронный ресурс]. URL: auts.esrae.ru/4-78 (дата обращения: 26.05.2013).

[24] Варламов О.О., Сергушин Г.С., Елисеев Д.В., Адамова Л.Е., Майборода Ю.И., Антонов П.Д., Чибирова М.О. О миварном подходе к моделированию процессов понимания компьютерами смысла текстов, речи и образов. Новые возможности расширения границ автоматизации умственной деятельности человека. // Автоматизация и управление в технических системах. - 2013. - № 2(4); C. 30-45. [Электронный ресурc]. URL: auts.esrae.ru/4-80 (дата обращения: 26.05.2013).

[25] Сергушин Г.С., Варламов О.О., Чибирова М.О., Елисеев Д.В., Муравьева Е.А. Исследование возможностей информационного моделирования сложных систем управления технологическими процессами на основе миварных технологий // Автоматизация и управление в технических системах. - 2013. - № 2(4); С. 46-60. [Электронный ресурc]. URL: auts.esrae.ru/4-81 (дата обращения: 26.05.2013).

[26] Варламов О.О., Адамова Л.Е., Елисеев Д.В., Майборода Ю.И., Антонов П.Д., Сергушин Г.С., Чибирова М.О. Комплексное моделирование процессов понимания компьютерами смысла текстов, речи и образов на основе миварных технологий // Искусственный интеллект. - 2013. - № 4. - С. 15-27.

[27] Чибирова М.О., Сергушин Г.С., Варламов О.О., Елисеев Д.В., Хадиев А.М. и др. Реализация общедоступного миварного универсального решателя задач на основе адаптивного активного логического вывода с линейной сложностью и облачных технологий // Искусственный интеллект. - 2013. - № 3. - С. 512-523.

[28] Сергушин Г.С., Варламов О.О., Чибирова М.О., Елисеев Д.В., Муравьева Е.А. Информационное моделирование сложных автоматизированных систем управления технологическими процессами на основе миварных технологий // Искусственный интеллект. - 2013. - № 3. - С. 126-138.

[29] Варламов О.О., Чибирова М.О., Сергушин Г.С., Елисеев Д.В. Практическая реализация универсального решателя задач «УДАВ» с линейной сложностью логического вывода на основе миварного подхода и «облачных» технологий // Приборы и системы. Управление, контроль, диагностика. - 2013. - № 11. - С. 4555 .

[30] Остроух А.В. Системы искусственного интеллекта в промышленности, робототехнике и транспортном комплексе: монография / A.B. Остроух Красноярск: Научно-инновационный центр, 2013. - 326 с. - ISBN 978-5-906314$10-9$. 